\title{
Dial a Doctor: Improving lines of communication on the acute admissions unit.
}

Fionnuala Schwartz

St Barts NHS Trust

\begin{abstract}
The Royal London Hospital operates a system of 'specialty take triage', meaning that multiple teams provide patient care on the hospital's Acute Admission Unit (AAU). The aim of this project is to ensure the medical team for each patient can be promptly and easily contacted.

An initial staff survey and engagement with key stakeholders assessed the baseline situation and guided a series of interventions, including the creation of ward whiteboards and funding for more portable phones. During further improvement cycles representatives from each profession were recruited to promote the new system, working groups held, and presentations made at staff meetings. The staff survey was repeated to gauge improvement.
\end{abstract}

The initial survey of 33 staff revealed that there was no reliable system for ascertaining the specialty team to which a patient had been allocated or information on how to contact them. $39 \%$ of respondents reported experiencing a situation they felt was unsafe.

Following the initial interventions, 25 staff responded to a second survey. $96 \%$ responded that the new system had made it easier to contact the correct medical team. The percentage of staff reporting difficulty knowing which medical team to contact most or everyday reduced from $66 \%$ to $32 \%$. The percentage of those reporting difficulty contacting that team most or everyday reduced from $36 \%$ to $12 \%$. There were 22 respondents to the survey following the second round of intervention. Less than $5 \%$ of staff reported difficulty most or everyday in both identifying the correct medical team and contacting them.

There has been marked improvement in the ease of identifying and contacting the medical teams. This project is ongoing, recognising that further progress is required to ensure patient safety.

\section{Problem}

The Royal London Hospital's Acute Admissions Unit (AAU) uses a specialty take triage system meaning that patients on the ward are cared for by different medical specialties.

There was no clear system for staff to easily identify:

1. which medical specialty a patient had been triaged to

2. which AAU doctor was assigned to that team

3. how to contact that doctor/team

This problem was readily identified by staff on the ward and there were concerns that patient safety was being compromised as a result.

\section{Background}

The Royal London Hospital's AAU has over 50 beds and a large team of staff. The unit operates a specialty take triage system, allocating admissions to the most appropriate medical teams as early as possible. Patients on the unit may be cared for by the Take team (who assess new patients on their day of admission), the Posttake team (who review the patients admitted the day prior), or one of many other specialty teams. For staff on the unit, knowing which team was responsible for each patient and how to contact them was challenging as no clear system existed for disseminating this information. For example, a staff nurse on the ward looking after 6 patients could have to liase with up to 6 different medical teams. Before this project began the nurse had no reliable method of knowing which team to contact or how to do so. Adding to the confusion, the AAU junior doctors would rotate between take, posttake, and specialty teams on a weekly basis. Therefore the doctor who was the first point of contact for Health Care of the Elderly patients one week would be covering the new Take patients the following week.

Knowledge was gathered locally to see what systems were working on other wards in the hospital. Bedboards were a common intervention for clearly presenting logistical information about patients on the ward, including the team responsible for them. These were most successful when undated regularly by staff on the ground. Many multidisciplinary team (MDT) members preferred doctors to use portable phones instead of bleeps and highlighted that problems occurred when teams did not have individual contact numbers.

\section{Baseline measurement}


A survey, designed alongside MDT members, was completed by 33 ward staff, excluding doctors. Initial results revealed that two thirds experienced difficulty knowing which medical team was looking after their patients most or everyday. The remaining third reported this to be a problem some days. When asked how often they encountered difficulty contacting that medical team, over one third of staff answered most or everyday.

Baseline data confirmed that there was no reliable system for ascertaining the specialty team to which a patient had been allocated or information on how to contact them. Most concerning was that $39 \%$ of respondents had experienced a situation which they felt was unsafe as a result, compromising patient safety. For example, one nurse reported that when looking after an unwell patient their treatment was delayed because he/she was unable to contact doctors. After a number of failed attempts another doctor, who did not know the patient, ended up attending to them.

See supplementary file: ds3967.doc - "survey"

\section{Design}

An initial staff survey and engagement with key stakeholders assessed the baseline situation and guided a series of interventions, including the creation of ward whiteboards and funding for more portable phones. The key stakeholders were ward sisters, the AAU clinical lead, AAU consultants, and junior doctors. All were motivated to become involved due to widespread frustration with the inefficiency of the current system and moreover the evidence that patient safety was being compromised.

Our ward whiteboard was designed to show the specialty team to which each patient had been allocated, the named junior doctor for that team, and their contact number. By presenting the baseline data to department leads we were able to secure funding for more portable phones. Interventions were publicised at ward meetings and representatives were recruited from different MDT groups to publicise the whiteboard by word of mouth. The staff survey was repeated in each cycle to measure improvement and identify the ongoing barriers to success. The project so far, which has involved three PDSA cycles was completed over a four month period. In order to ensure sustainability and ongoing improvement, we have made efforts to widened staff involvement and handed over the project to the new cohort of junior doctors.

\section{Strategy}

Cycle 1

Plan: To assess the current situation and understand the factors contributing to the problem

Do: Survey designed alongside MDT and then completed by ward staff $(n=33)$

Study: $39 \%$ of staff had experienced a situation which they felt was unsafe because they could not contact the medical team. Major barriers: No reliable system for ascertaining which specialty team a patient has been allocated to and not enough portable phones or bleeps

Act: Proceeded with urgency to engage with key stakeholders and to form the project team which included - Ward Sisters, Junior Doctors, Consultants, \& AAU leads

Cycle 2

Plan: To make it easier for staff to identify which medical team is looking after their patients and to make it easier to contact that medical team.

Do: Ward whiteboard implemented, funding secured for more portable phones and interventions publicised at ward meetings. Staff survey repeated $(n=25)$ and staff focus groups held to explore ongoing barriers to improvement.

Study: $96 \%$ responded that the new system had made it easier to contact the correct medical team. The percentage of staff reporting difficulty knowing which medical team to contact most or everyday reduced from $66 \%$ to $32 \%$. The percentage of those reporting difficulty contacting that team most or everyday reduced from $36 \%$ to $12 \%$. Ongoing barriers: Whiteboard not up to date, not enough phones and contact numbers not clearly documented

Act: Representatives recruited from different MDT groups to publicise the whiteboard by word of mouth. Meeting of project leads to plan next cycle.

Cycle 3

Plan: To make it easier for staff to identify which medical team is looking after their patients and to make it easier to contact that medical team.

Do: More phones and charging units secured \& all clearly labelled. Working groups with staff groups culminating in pledges. e.g. F1s agreed to update whiteboard each morning \& ward sisters agreed to update throughout the day. Survey repeated $(n=22)$

Study: Less than $5 \%$ of staff reported difficulty most or everyday in both identifying the correct medical team and contacting them.

Act: Staff informed of further improvements and handover of project to the new rotation of Junior Doctors

\section{Results}

Following the implementation of the ward whiteboard and the other initial interventions 25 staff responded to a second survey. $96 \%$ responded that the new system had made it easier to contact the correct medical team. The percentage of staff reporting difficulty knowing which medical team to contact most or everyday reduced from $66 \%$ to $32 \%$. The percentage of those reporting difficulty contacting that team most or everyday reduced from $36 \%$ to $12 \%$. There were 22 respondents to the third survey. Less than $5 \%$ of 


\section{BMJ Quality Improvement Reports}

staff reported difficulty most or everyday in both identifying the correct medical team and contacting them.

See supplementary file: ds3968.jpg - "whiteboard"

\section{Lessons and limitations}

I have learnt that the importance of engaging key stakeholders early cannot be underestimated. By identifying the right people and forming a dedicated working group the chance of improvement and sustained change is maximised. Given more time and the chance to apply for funding I would have been interested to use technology to make further innovations in this area - for example, exploring the possibility of an electronic bedboard.

The lessons learnt from this project have been disseminated through word of mouth by those involved and presented at a national conference. A recommendation for others who plan to start a project to improve communication in their clinical areas is to collect meaningful data from the outset. We designed our staff questionnaire alongside other MDT members so that its wording was clear and the questions relevant. From the results we were able to gain an idea not only of the baseline situation but also the major barriers to improvement as perceived by the staff on the ground. Another consideration is that when you implement a whiteboard or alternate message board in your clinical area there needs to be a plan made for who will update this. It can be difficult to motivate clinical staff to take on responsibilities on top of their already busy workload. A contingency plan is required for when the person/s are away or busy and the system needs to hold up to a "worst case scenario" busy working day on the ward.

\section{Conclusion}

The project has been successful in delivering a marked improvement in the ease of identifying and contacting medical teams. However the project is ongoing, recognising that further progress is required to ensure patient safety.

\section{References}

1. McKnight L, Stetson P, Bakken S, Curran C, Cimino J. Perceived Information Needs and Communication Difficulties of Inpatient Physicians and Nurses. J Am Med Inform Assoc. 2002 Nov-Dec; 9(6 Suppl 1): s64-s69.

2. Kohn K, Corrigan J, Donaldson M (editors for the Committee on Quality of Health Care in America). To Err is Human: Building a Safer Health System. Institute of Medicine. National Academy Press, 1999.

3. Taylor M, McNicholas M, Nicolay C, Darzi A, Bell D, Reed J. Systematic review of the application of the plan-do-study-act method to improve quality in healthcare. BMJ Qulaity and Safety. 2013. Acessible online at: http://qualitysafety.bmj.com/content/early/2013/09/11/bmjqs2013-001862.full

\section{Declaration of interests}

none

\section{Acknowledgements}

Dr Louella Vaughan, Dr Mona Waterhouse \& all staff on the Royal London Hospital's AMU. 